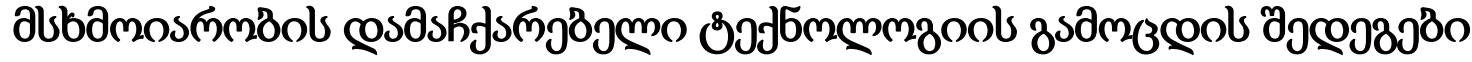

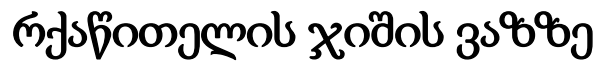

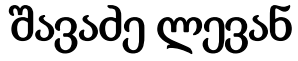

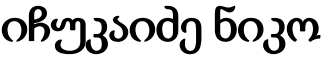

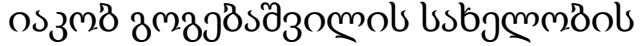

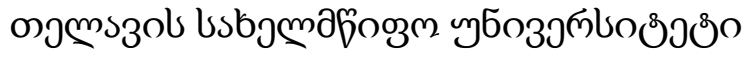

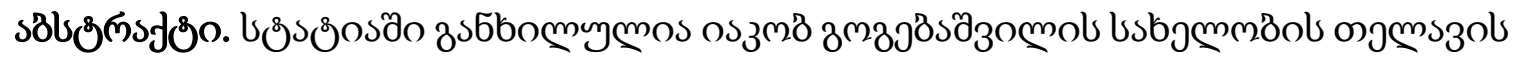

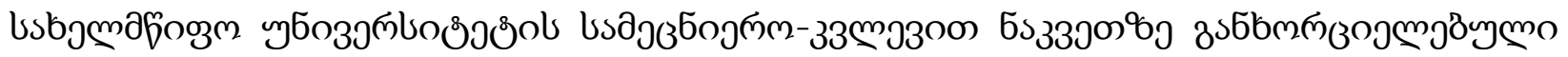

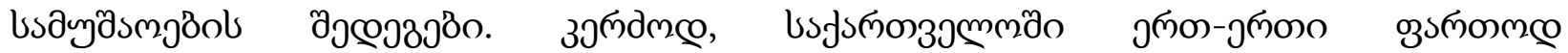

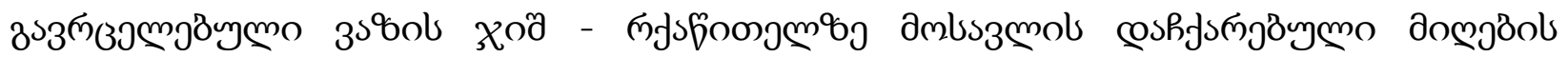

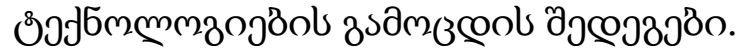

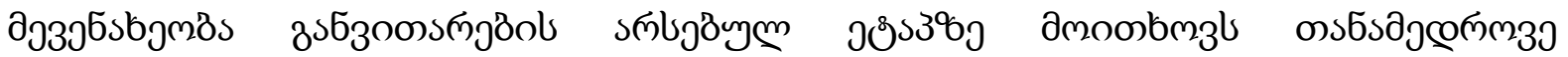

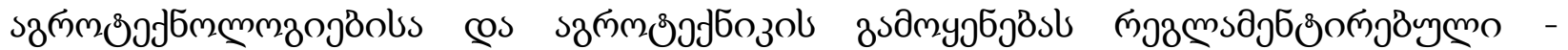

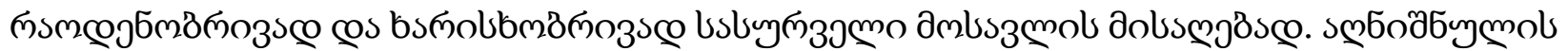

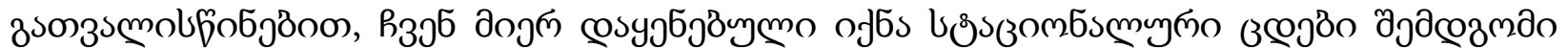

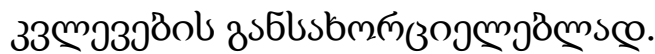

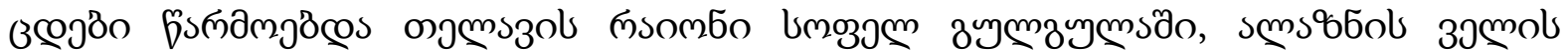

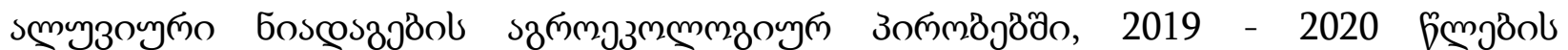

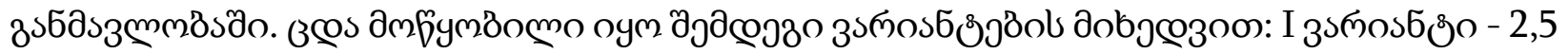

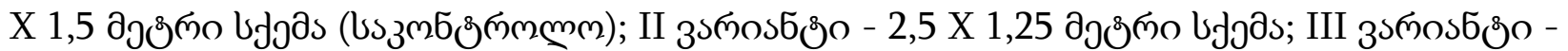

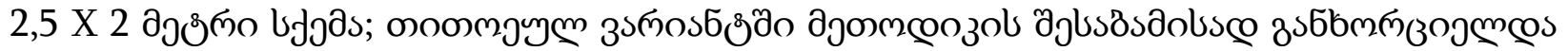

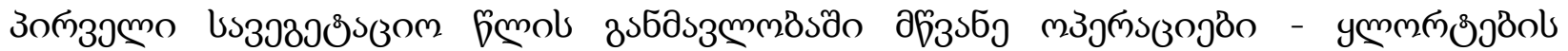

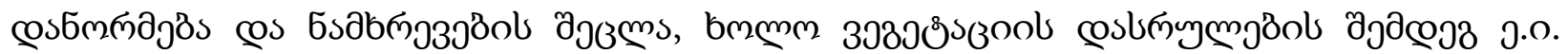

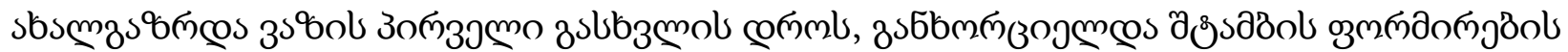

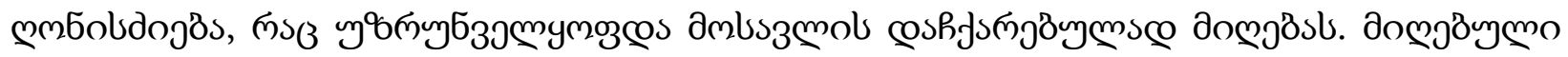

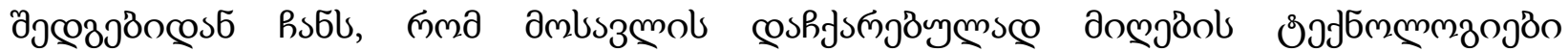

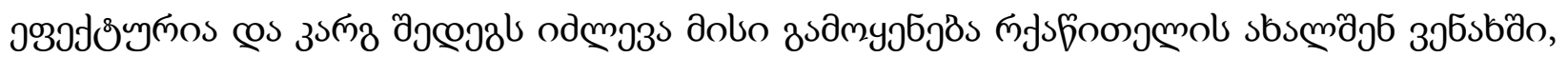

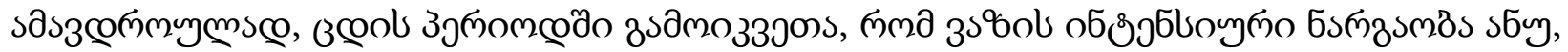

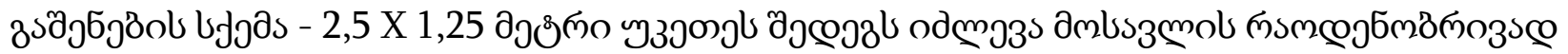
zs

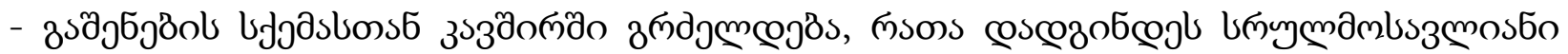

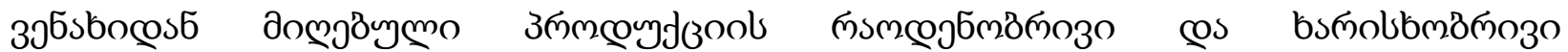

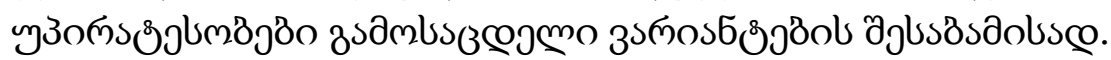




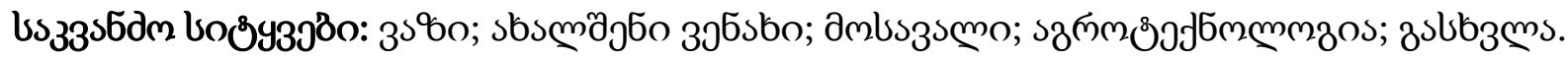

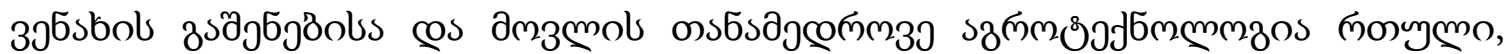

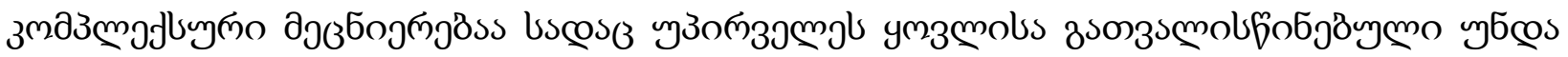

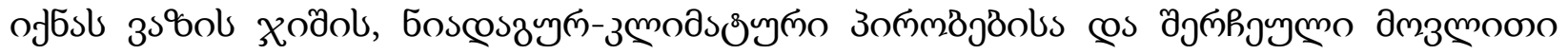

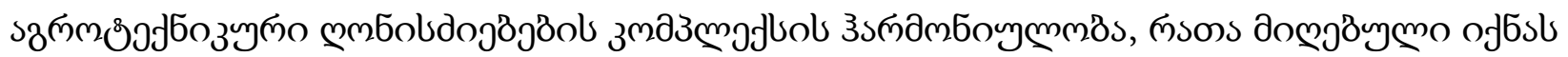

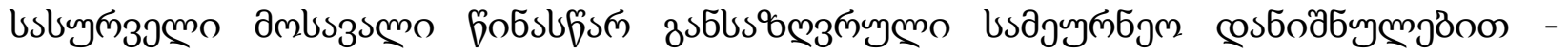

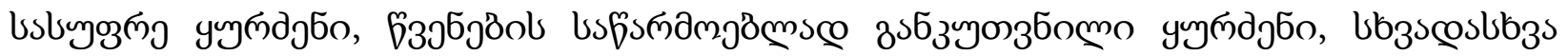

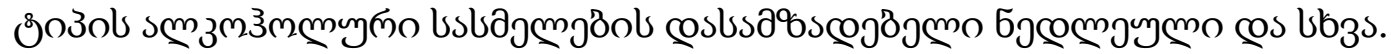

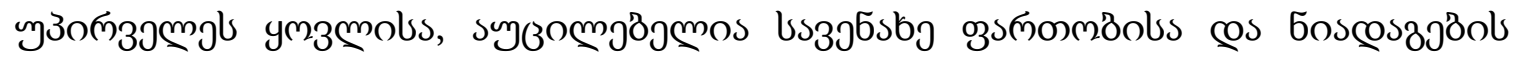

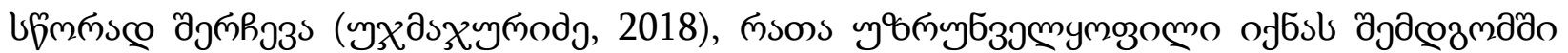
3s\%ol bsluy

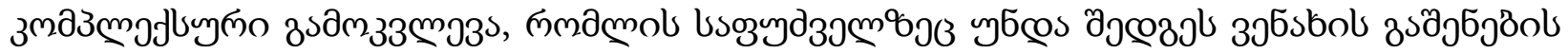

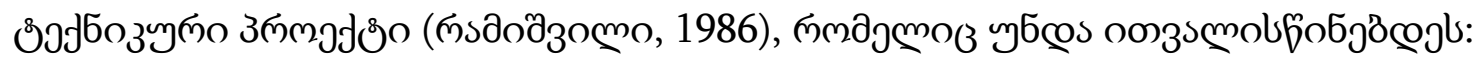

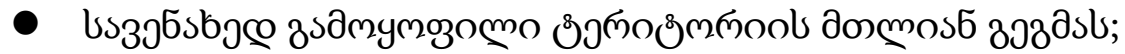

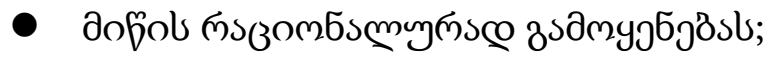

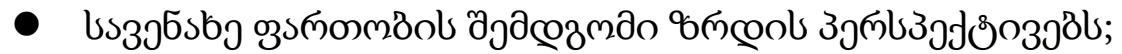

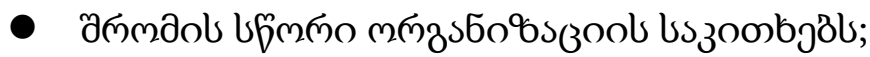

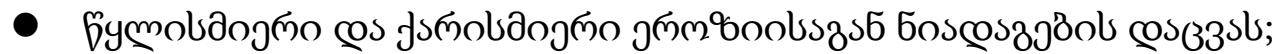

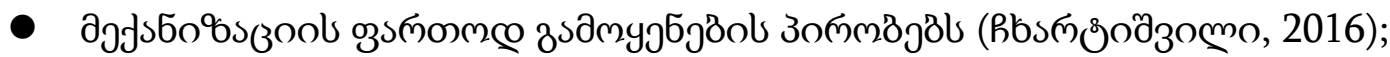

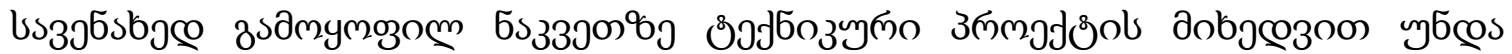

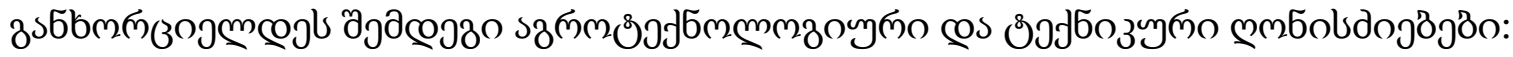

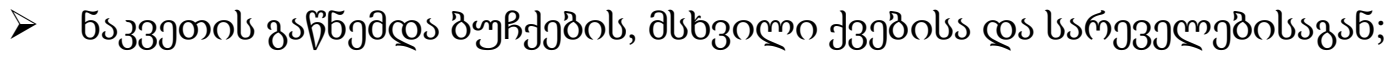

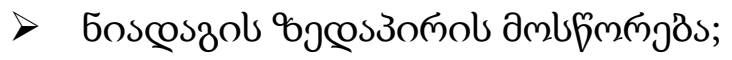

$>$ usøfy

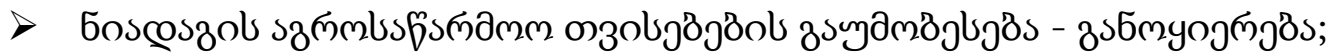

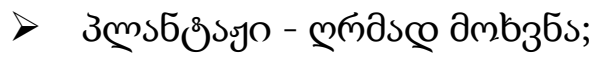

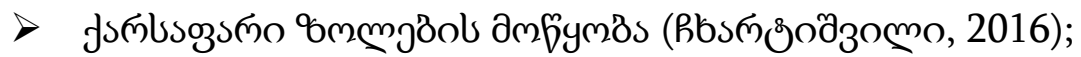

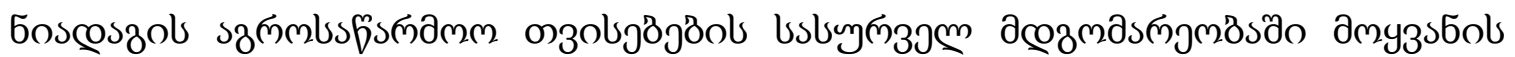

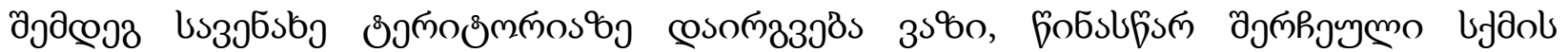
дobjœ3

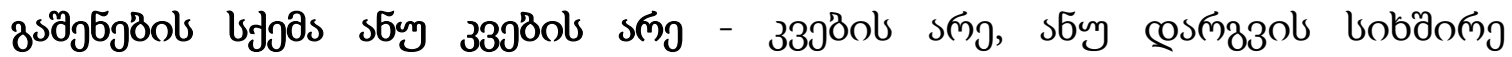

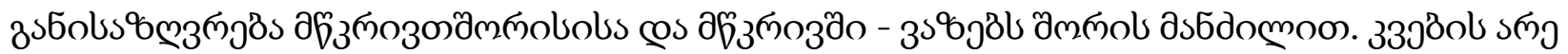

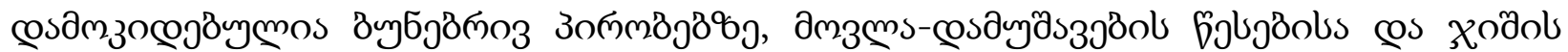

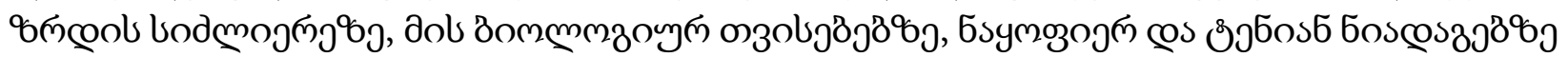
3

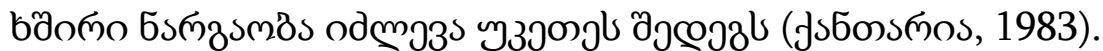

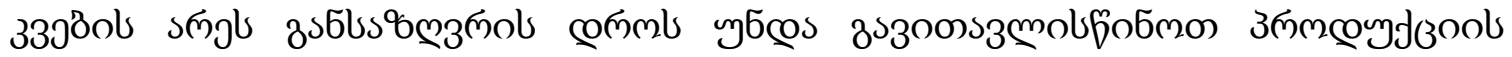




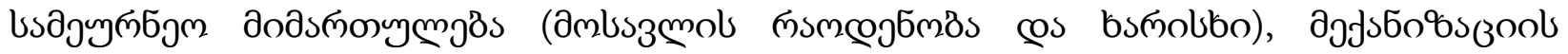

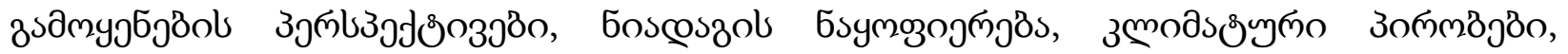

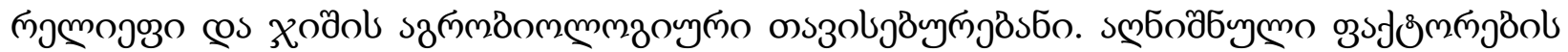

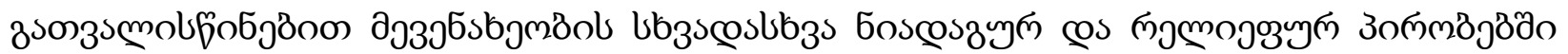

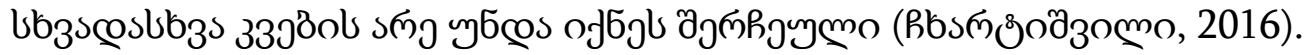

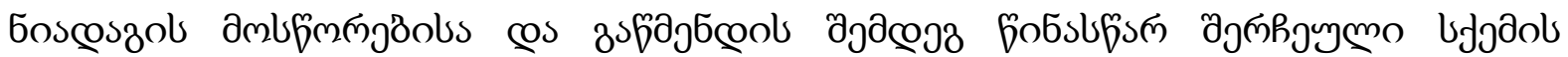

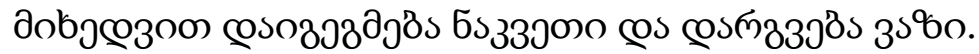

\section{ззмлзоl дјомеоз}

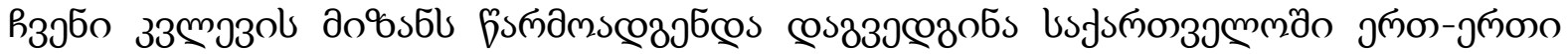

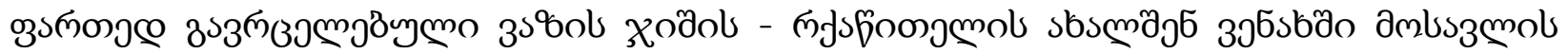

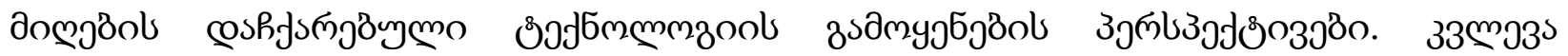

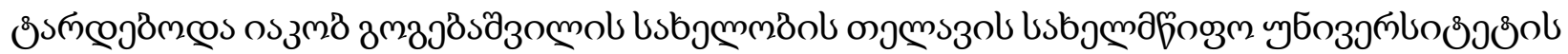

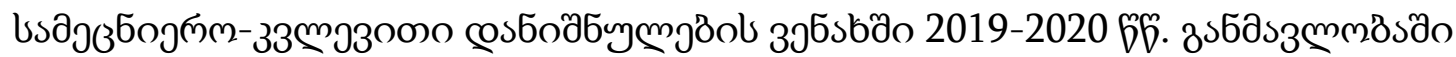

2019 6ృ

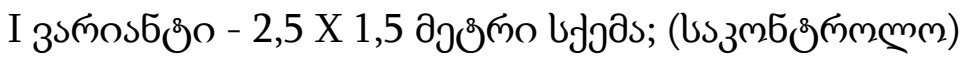

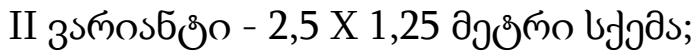

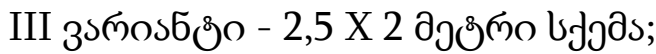

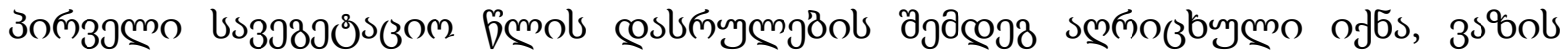

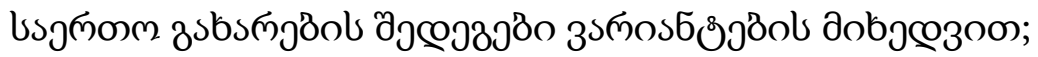

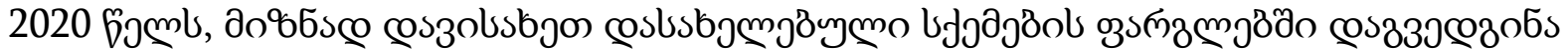

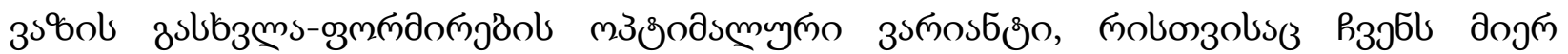

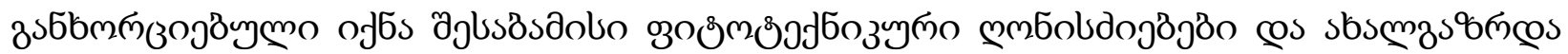
3

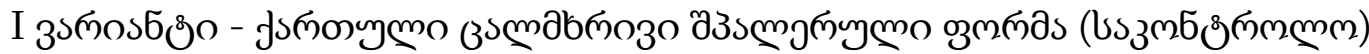

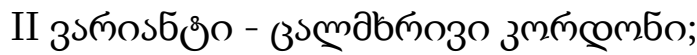

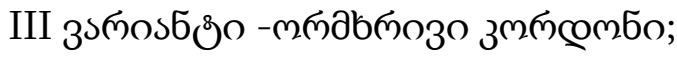

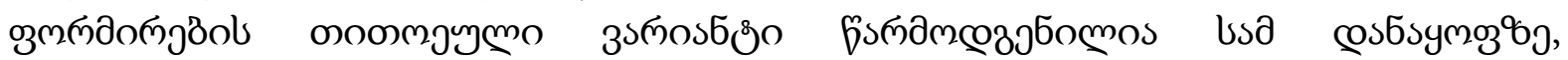

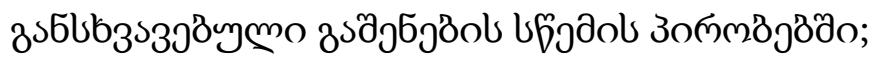

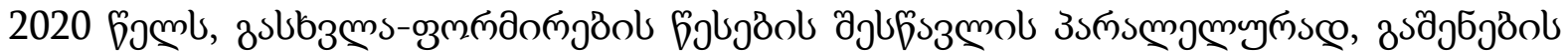

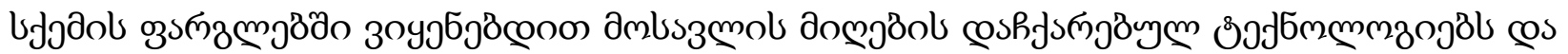

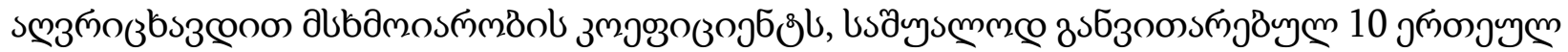

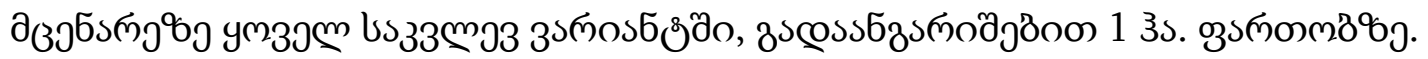

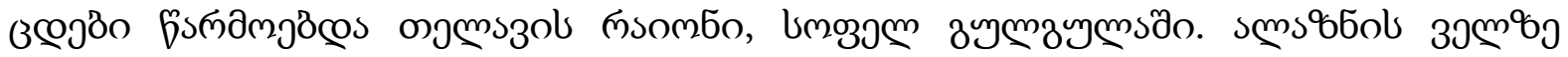

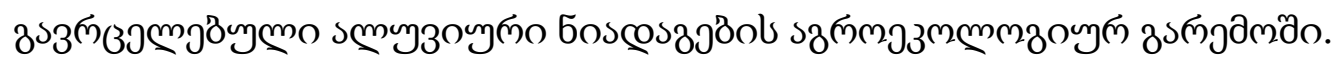

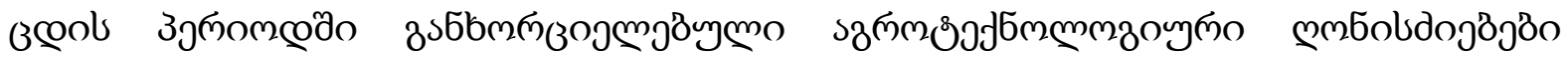

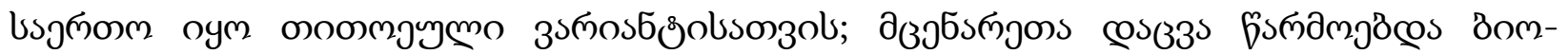

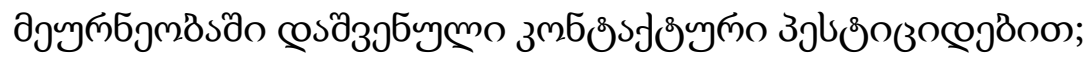

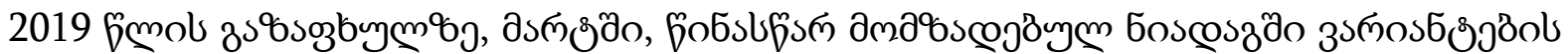

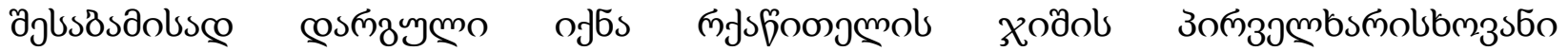

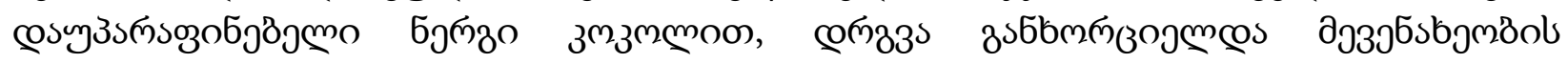

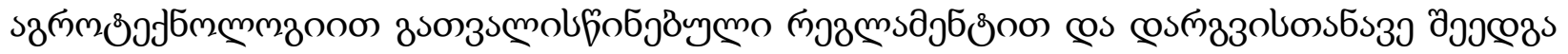


łozm;

obromo 1

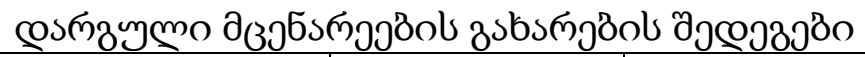

\begin{tabular}{|c|c|c|c|}
\hline 3కగnంs6తిం & $\begin{array}{r}\text { bygm } \\
\text { cosmaymo }\end{array}$ & asobsms & $\begin{array}{l}30 \text { m } \\
\text { asobsms }\end{array}$ \\
\hline 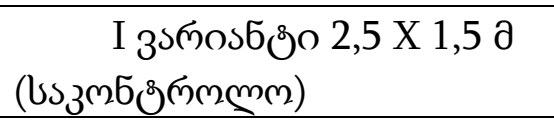 & 132 & 119 & 13 \\
\hline 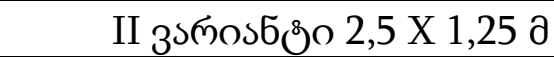 & 160 & 144 & 16 \\
\hline 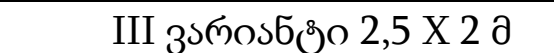 & 100 & 98 & 2 \\
\hline
\end{tabular}

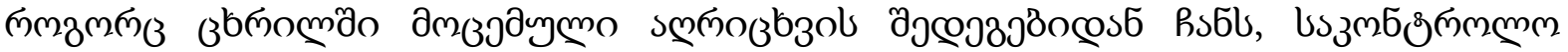

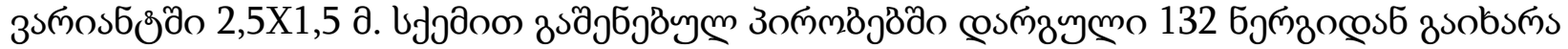

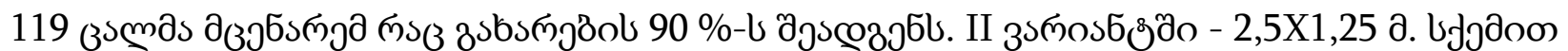

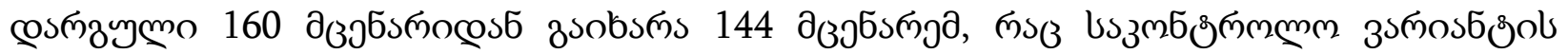

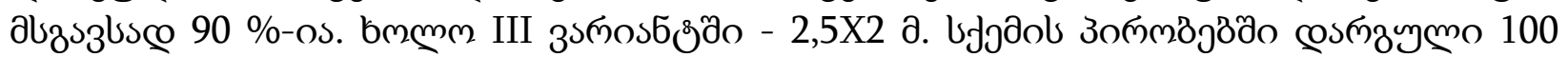

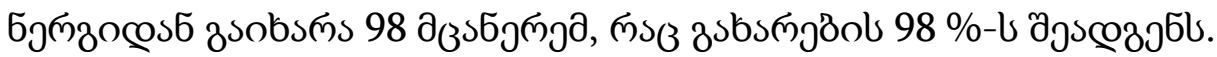

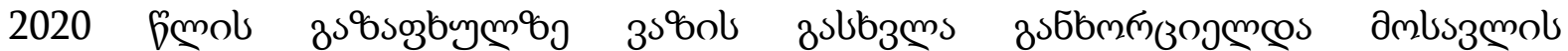

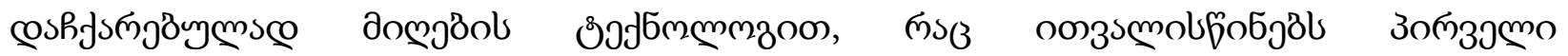

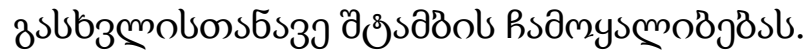

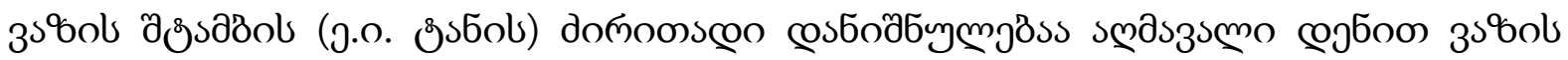

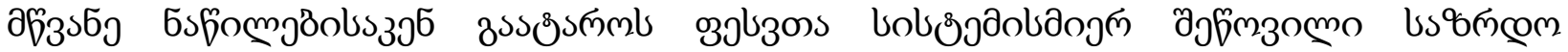

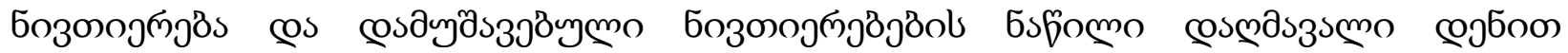

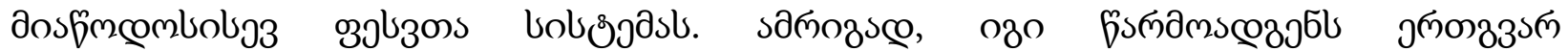

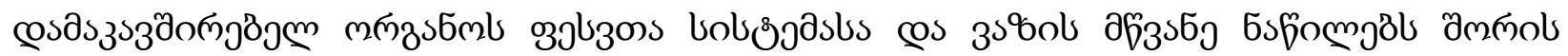

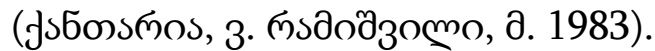

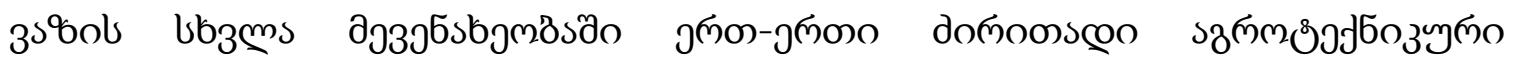

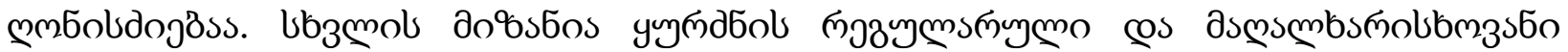

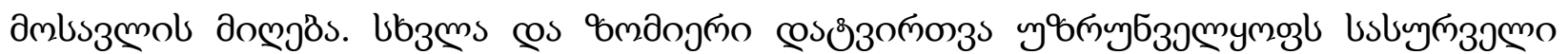

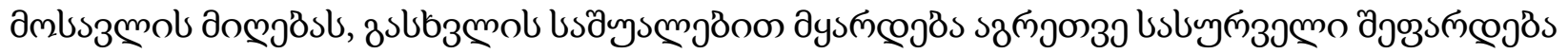

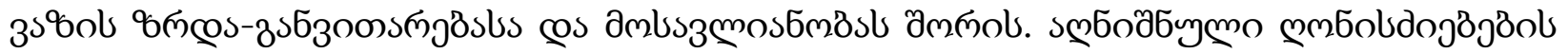

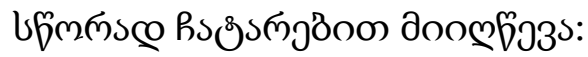

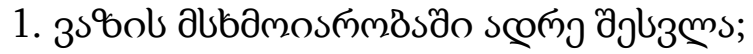

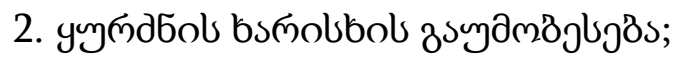

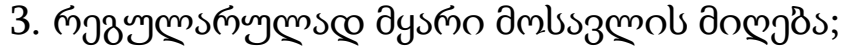

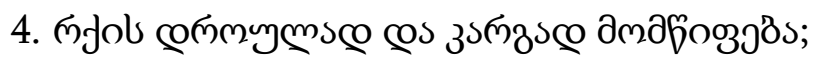

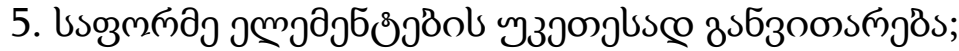

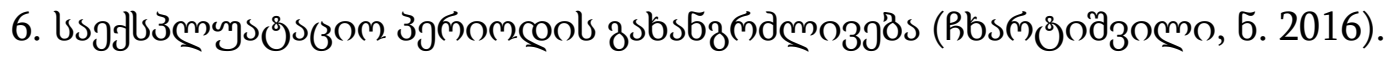

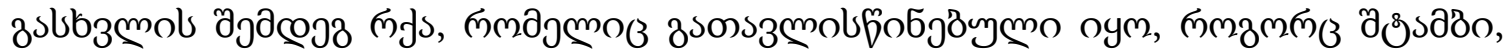

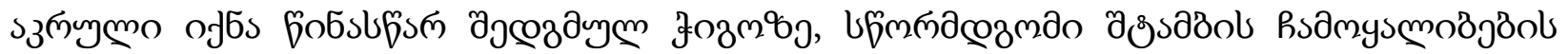

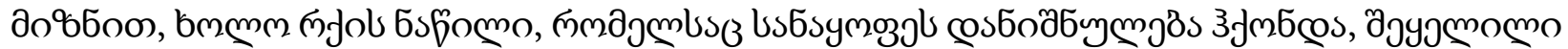




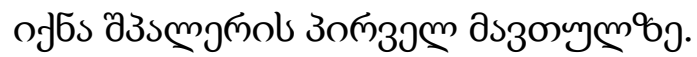

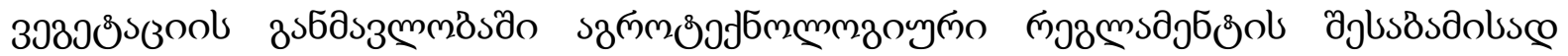

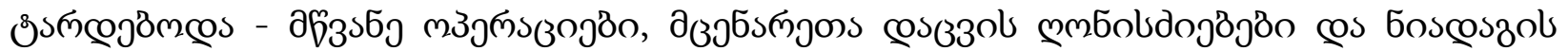

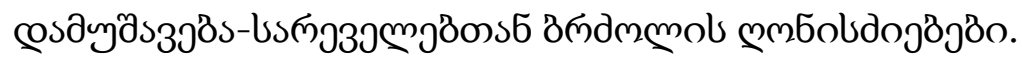

उbromo 2

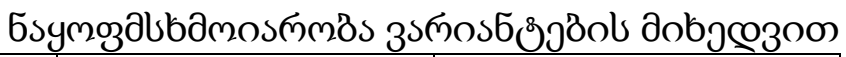

\begin{tabular}{|c|c|c|c|}
\hline \multirow{2}{*}{ 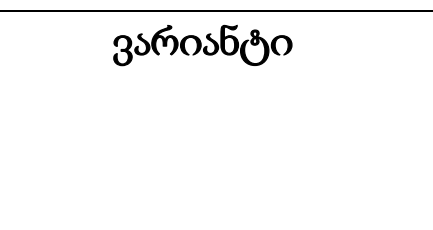 } & uszyysmme & usayzsmme & abbamosmmbs \\
\hline & 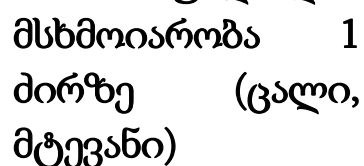 & 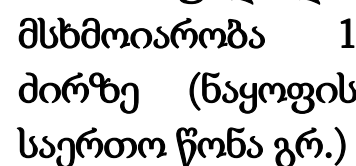 & 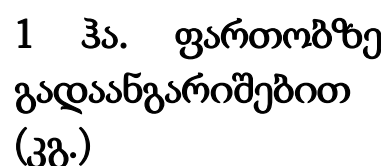 \\
\hline 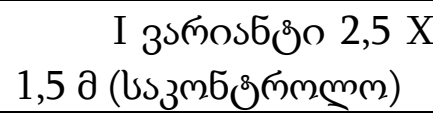 & 2 & 400 & 1056 \\
\hline 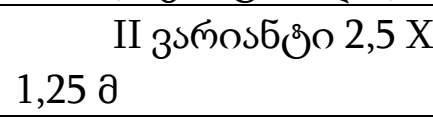 & 2 & 400 & 1280 \\
\hline 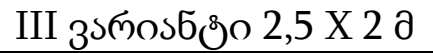 & 2 & 400 & 800 \\
\hline
\end{tabular}

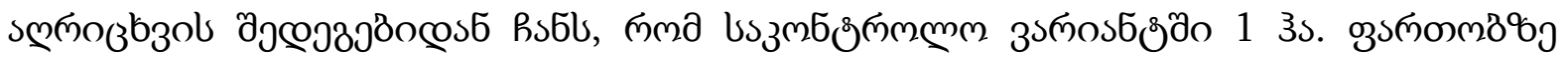

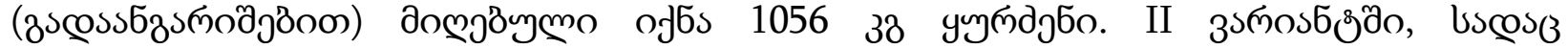

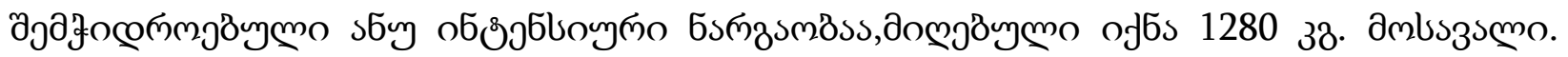

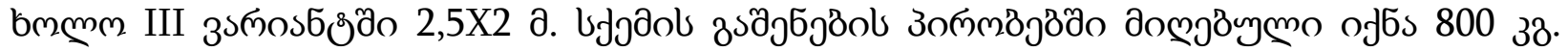
dmbšssmo.

\section{cosb336j8о:}

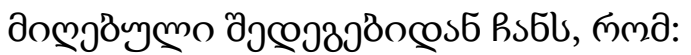

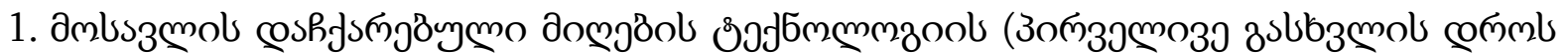

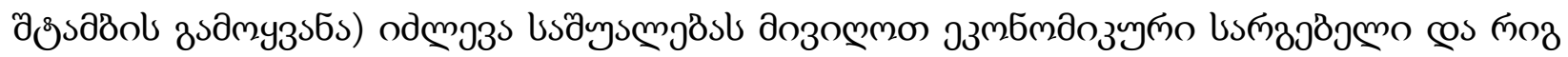

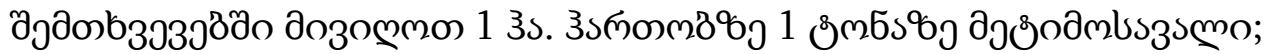

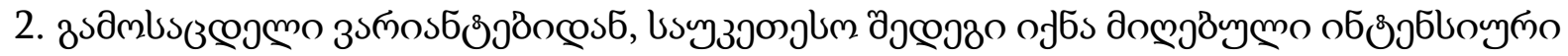

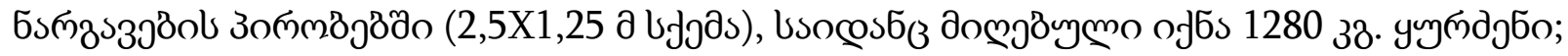

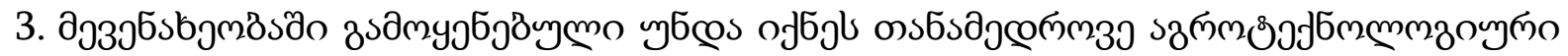

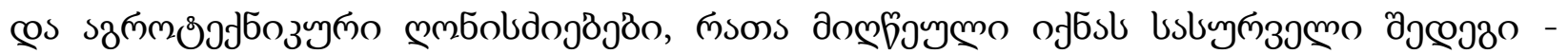

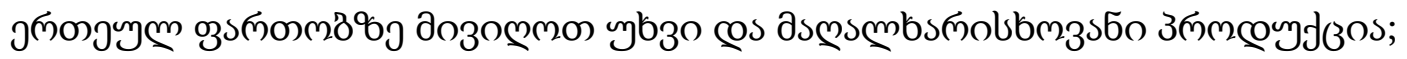

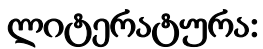

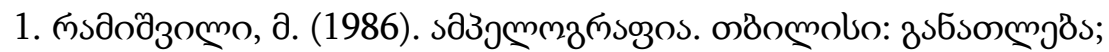

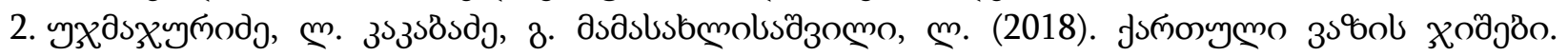

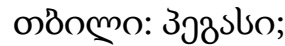

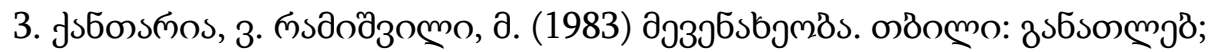

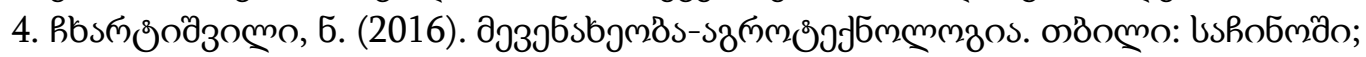




\title{
Test Results of the Technology Accelerating the Fructification of Rkatsiteli Vine
}

\author{
Shavadze Levan \\ Ichukaidze Niko \\ Iakob Gogebashvili Telavi State Universit
}

\begin{abstract}
The article discusses the results of the work carried out on the scientific - research plot of land of Iakob Gogebashvili Telavi State University, in particular, the test results of the technologies accelerating the harvest on one of the most widespread vine species in Georgia such as Rkatsiteli.

The experiments were carried out in the village Gulgula, Telavi district, in the agro ecological conditions of the alluvial soils of the Alazani Valley during the years of $2019-2020$. The experiment was arranged according to the following options: Option I - 2,5 X 1,5 meter scheme (control); Option II - 2,5 X 1,25 meter scheme; Option III - 2,5 X 2 meter scheme; According to the methodology green operations were carried out in each option during the first vegetation year, such as normalizing sprouts and removing suckers and after the completion of the vegetation, i.e. during the initial pruning of a young vine there was carried out the formation of a stem, which provided to speed up harvesting. The obtained results show that the technologies that speed up harvesting are effective and their usage provides good results in the vineyard planted with Rkatsiteli. At the same time, during the experiment it became clear that intensive vine crop, i.e. planting scheme $2,5 \times 1,25 \mathrm{~m}$. provides better result in terms of quantitative growth of the crop per unit area in the planted vineyard.
\end{abstract}

Key Words: vine; planted vineyard; crop; agrotechnology; pruning 